Final Progress Report

Project Title: Image-guided Non-invasive Ultrasonic Thrombolysis Using

Histotripsy

PI: Zhen Xu, University of Michigan

12/9/2013 - 3/31/2015

\title{
- Summary of Progress
}

Histotripsy, a novel, non-invasive therapeutic technique, employs controlled pulsed cavitational ultrasound for tissue ablation and is currently being evaluated for thrombolysis and palliation of congenital heart disease. Some forms of therapeutic ultrasound cause intravascular hemolysis. Hemolysis due to histotripsy has not been previously evaluated. This study aimed to investigate the extent and consequences of hemolysis caused by histotripsy in vivo. Free flowing blood in the porcine femoral vein was treated with histotripsy therapy in 11 animals with systemic heparinization and 11 animals without heparin. Serum and hemodynamic measurements were obtained at baseline (0), 2, 5, 10, 15, and $30 \mathrm{~min}$, as well as 48-72 hours post-procedure. Of the 11 non-heparin treated animals, 5 died during or immediately following Histotripsy, while all animals in the heparin group survived (non-heparin group mortality $45 \%$ vs. heparin group mortality $0 \%, p<0.05)$. Among all animals, serum hematocrit decreased slightly following histotripsy (32.5 \pm 3.6 to $29.4 \pm 4.2 \%, p<0.001)$. There was a significant increase in free hemoglobin $(6.2 \pm 4.6$ to $348 \pm 100 \mathrm{mg} / \mathrm{dL}, \mathrm{p}<0.001)$ and LDH $(365 \pm 68$ to $722 \pm 85 \mathrm{U} / \mathrm{L}, \mathrm{p}<0.001$ ). Right ventricle systolic pressure (RVSP) also increased (23.2 \pm 7.2 to $39.7 \pm 12 \mathrm{mmHg}, p<0.001$ ). After 48 to 72 hours, hematocrit remained slightly decreased, and LDH remained slightly increased, compared to baseline, though free hemoglobin nearly returned to baseline. These results show that histotripsy applied to the circulating blood volume is associated with high mortality in non-heparinized animals, but is generally safe with systemic heparinization. A transient increase in free hemoglobin was observed with no clinically significant reduction in total hematocrit. There is also a transient increase in RVSP.

In previous histotripsy-mediated thrombolysis studies, cavitation clouds were generated using multi-cycle pulses and tended to form on vessel wall. To avoid potential cavitational damage to vessel wall, a new histotripsy approach, termed Microtripsy, has been recently discovered where cavitation is generated via an intrinsic-threshold mechanism using single-cycle pulses. We hypothesize that microtripsy can generate and confine cavitation in vessel lumen without contacting vessel wall, which results in recanalization within clot and potentially eliminating vessel damage. To test our hypothesis, microtripsy was investigated for clot recanalization in an in vitro flow model. Clots were formed inside a vessel phantom (6.5 mm inner diameter) in line with a flow system. Microtripsy was applied by a $1-\mathrm{MHz}$ transducer at a pulse repetition frequency of $50 \mathrm{~Hz}$ with a peak negative pressure (P-) of $30 \mathrm{MPa}$ or $36 \mathrm{MPa}$. To create a flow channel through a clot, the cavitation focus was scanned through the clot at an interval of 0.3 or $0.7 \mathrm{~mm}$. The treated clots were 3D-scanned by a $20-\mathrm{MHz}$ ultrasound probe to quantify the channels. Restored flow rates were measured and clot debris particles generated from the treatments were analyzed. In all treatments, cavitation cloud was consistently generated in the center of the vessel lumen without contacting the vessel wall. After each treatment, a flow channel was successfully generated through and completely confined inside the clot. The channels had a diameter up to $60 \%$ of the vessel diameter with restored flow up to $500 \mathrm{~mL} / \mathrm{min}$. The debris particles were small with over $99.9 \%<10 \mu \mathrm{m}$ and the largest at $153 \mathrm{um}$. Each clot $(2 \mathrm{~cm}$ long) was recanalized within $7 \mathrm{~min}$. The size of the flow channels increased by using higher P-and 
was significantly larger by using the $0.3 \mathrm{~mm}$ scan interval than those using $0.7 \mathrm{~mm}$. The results in this study show the potential of this new microtripsy thrombolysis method for fast, precise, and effective clot recanalization, minimizing risks of vessel damage and embolism.

Real-time quantitative feedback is highly desired during histotripsy thrombolysis treatment to monitor the progress of clot fractionation. B-mode ultrasound imaging during histotripsy therapy can easily visualize cavitation due to the hyperechogenicity of the bubble cloud and provide precise targeting guidance, but its ability to serve as a quantitative therapy feedback is limited. Bubble-induced color Doppler (BCD) monitors the motion following cavitation generated by each histotripsy pulse, which has been shown in gel and ex vivo liver tissue to be correlated with histotripsy fractionation. We investigated the potential of BCD to quantitatively monitor histotripsy thrombolysis in real-time. To visualize clot fractionation, transparent three-layered fibrin clots were developed. Results show a coherent motion followed the cavitation generated by each histotripsy pulse with a push and rebound pattern. The temporal profile of this motion expanded and saturated as the treatment progressed. A strong correlation existed between the degree of histotripsy clot fractionation and two metrics extracted from BCD: time of peak rebound velocity (tprv) and focal mean velocity at a fixed delay $\left(\mathrm{V}_{\mathrm{f}, \text { delay }}\right)$. The saturation of clot fractionation (i.e., treatment completion) matched well with the saturations detected using $t_{P R V}$ and $V_{f, d e l a y}$. The mean Pearson correlation coefficients between the progressions of clot fractionation and the two BCD metrics were $93.1 \%$ and $92.6 \%$ respectively. To validate the BCD feedback in in vitro clots, debris volume from histotripsy thrombolysis were obtained at different therapy doses and compared with

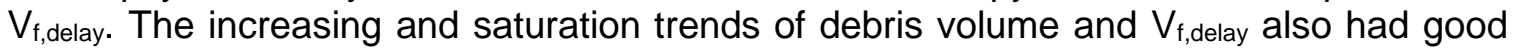
agreement. Finally, a real-time BCD feedback algorithm to predict complete clot fractionation during histotripsy thrombolysis was developed and tested. This work demonstrated the potential of BCD to monitor histotripsy thrombolysis treatment in realtime.

\section{- Publications}

Published and In Press

1. Vlaisavljevich E, Maxwell A, Warnez M, Johnsen E, Cain CA, Xu Z. Histotripsyinduced cavitation cloud initiation thresholds in tissues of different mechanical properties. IEEE Trans Ultrason Ferroelectr Freq Control. 2014;61(2):341-52.

2. Vlaisavljevich E, Lin KW, Warnez MT, Singh R, Mancia L, Putnam AJ, Johnsen E, Cain C, Xu Z. Effects of tissue stiffness, ultrasound frequency, and pressure on histotripsy-induced cavitation bubble behavior. Phys Med Biol 2015;60(6):2271-92.

3. Zhang X, Miller RM, Lin KW, Levin AM, Owens GE, Gurm HS, Cain CA, Xu Z. Realtime Feedback of Histotripsy Thrombolysis Using Bubble-induced Color Doppler. Ultrasound Med Biol. 2015, Epub.

4. Devanagondi R, Zhang X, Gurm HS, Levin AM, Xu Z, Owens GE. The hemodynamic and hematological effects of histotripsy. Journal of vascular and interventional radiology. In Press. 
5. Zhang X, Owens GE, Gurm H, Ding Y, Cain CA, Xu Z. Non-invasive Thrombolysis using Histotripsy beyond the "Intrinsic" Threshold (Microtripsy). IEEE Trans Ultrasonics Ferroelectrics Frequency Control. Conditionally accepted.

In Preparation

1. Zhang X, Jin L, Vlaisavljevich E, Cain CA, Owens GE, Gurm H, Xu Z. Non-invasive Thrombolysis using Microtripsy: A Parameter Study. In Preparation.

- Presentations

1. Zhang X, Owens G, Gurm HS, Ding Y, Cain C, Xu Z. Non-invasive Thrombolysis using Histotripsy beyond the "Intrinsic" Threshold (Microtripsy) International Society for Therapeutic Ultrasound Symposium; 2015 April 15-18; Utrecht, Netherlands.

2. Devanagondi R, Zhang X, Owens GE, Gurm HS, Levin AM, Xu Z. The hemodynamic and hematological effects of histotripsy. Focused Ultrasound Symposium; 2014 Oct 12-16; North Bethesda, MD.

3. Zhang X, Miller RM, Maxwell AD, Cain CA, Xu Z. Real-time Feedback of Histotripsy Thrombolysis using Bubble-induced Color Doppler. IEEE International Ultrasonics Symposium; 2014 Sep 3-6; Chicago, IL, USA.

4. Vlaisavljevich E, Warnez M, Singh R, Putnam A, Cain C, Xu Z. Response of Soft Tissue to Pulsed Cavitational Ultrasound Therapy - Histotripsy Paper presented at: National Congress on Theoretical and Applied Mechanics; June 15-20, 2014; Lansing, MI, USA.

5. Vlaisavljevich E, Warnez M, Johnsen E, Putnam A, Cain CA, Xu Z, Investigation of the Role of Tissue Mechanical Properties and Ultrasound Frequency in Histotripsyinduced Cavitation. International Symposium on Therapeutic Ultrasound; Apr 2-5, 2014, Las Vegas, NV, USA.

6. Devanagondi R, Xu Z, Zhang X, Ives K, Gurm H, Owens GE, editors. The hemodynamic and hematological effects of Histotripsy The Society for Cardiovascular and Angiography and Interventions Scientific Sessions; 2014 May 28-31; Las Vegas, NV.

\section{- Follow-on Funding}

Grant Title: Image-guided Non-invasive Ultrasonic Thrombrolysis Using Histotripsy

Grantor: NIH

Funding Mechanism: R01 renewal

Amount: $\$ 2,032,190.00$ (all to $U$ of Michigan)

Funding Period: 7/1/2014 - 6/30/2018

Notes:

This R01 renewal covers many aspects of the clinical translation of histotripsy for DVT and technical advancements to expand histotripsy thrombolysis for other applications.

\section{- Future Plan}

Based on the results from this grant, the ultrasound image guided histotripsy thrombolysis system will be redesigned to incorporate Microtripsy and bubble-induced color Doppler to increase the safety and efficacy of histotripsy thrombolysis system. The in vivo safety and efficacy of this new system will be determined through a comprehensive preclinical study in the porcine DVT model. In addition, together with HistoSonics, we (University of Michigan team) will actively seek industry partners to pursue the commercialization of the histotripsy thrombolysis technique. 